\title{
Entropy and topology for gravitational instantons
}

\author{
Stefano Liberati* \\ Scuola Internazionale Superiore di Studi Avanzati, Via Beirut 2-4, 34013 Trieste, Italy \\ Giuseppe Pollifrone ${ }^{\dagger}$ \\ Theory Division, CERN, CH-1211 Geneva 23, Switzerland \\ and Dipartimento di Fisica, Università di Roma 'La Sapienza," and INFN, Sezione di Roma, \\ Piazzale Aldo Moro 2, 00185 Roma, Italy
}

(Received 12 March 1997)

\begin{abstract}
In this work a relation between topology and thermodynamical features of gravitational instantons is shown. The expression for the Euler characteristic, through the Gauss-Bonnet integral, and the one for the entropy of gravitational instantons are proposed in a form that makes the relation between them self-evident. A new formulation of the Bekenstein-Hawking formula, where the entropy and the Euler characteristic are related by $S=\chi A / 8$, is obtained. This formula provides the correct results for a wide class of gravitational instantons described by both spherically and axially symmetric metrics. [S0556-2821(97)06020-7]
\end{abstract}

PACS number(s): 04.70.Dy, 04.20.Gz, 04.62.+v

\section{INTRODUCTION}

At the beginning of the 1970s an impressive series of theoretical results in general relativity were achieved, which led to an interpretation of some laws of black hole physics as thermodynamical ones [1-3]. Remarkably, it was found that black holes were endowed with an intrinsic entropy proportional to the horizon area, the so-called Bekenstein-Hawking entropy. Consistency for such a framework was subsequently achieved thanks to Hawking's discovery of black hole radiation [4], which results from the application of quantum field theory to such peculiar space-times. Soon after, thermodynamical aspects of black holes appeared more evident in the Euclidean path-integral approach [5]. If one considers the Einstein-Hilbert action plus a matter contribution in the generating functional of the Euclidean theory, one finds that the dominant contribution to the Euclidean path integral is given by gravitational instantons (i.e., nonsingular solutions of the Euclidean Einstein equations). In space-times with event horizons, this usually implies that metrics extremizing the Euclidean action are gravitational instantons only after removal of the conical singularity at the horizon [5]. A period must therefore be fixed in the imaginary time, which becomes a sort of angular coordinate. It is well known that Euclidean quantum field theory with periodic imaginary time is equivalent to a finite-temperature quantum field theory in Lorentzian space-time, where the temperature is the inverse of the imaginary time period. In such a way thermodynamics appears as a request of consistency of quantum field theory on space-times with Killing horizons, and in this sense we shall define such a thermodynamics as "intrinsic."

In Refs. [6,7] it has been shown that the BekensteinHawking law, $S=A / 4$, for black hole entropy fails for extremal ones. These objects were already considered "peculiar,' since their metric does not show any conical structure

\footnotetext{
*Electronic address: liberati@ sissa.it

†Electronic address: giuseppe.pollifrone@cern.ch
}

near their event horizon, so no conical singularity removal is required. The discovery of a zero entropy for extremal black holes, despite a nonzero area of the event horizon, made them even more important for the present investigation on the event horizon thermodynamics. In Ref. [6] it has been observed that the source of such a different behavior between extremal and nonextremal black holes is due to a change in the topological structure. In the latter case the presence of the event horizon is no longer associated with a nontrivial topology; the Euler characteristic indeed vanishes for extremal black holes, whereas it is different from zero for the nonextremal ones. All these considerations seem to suggest that extremal black holes should be considered as a rather different object from the nonextremal ones.

In this work we shall prove that Euler characteristic and gravitational entropy can be related in the same way in almost all known gravitational instantons endowed with event horizons. In particular, we shall show that the Euler characteristic and entropy have the same dependence on the boundaries of the manifold and we will relate them by a general formula. This formulation extends to a wide class of instantons, and in particular to the Kerr metric, the known results [8-11] about such a dependence.

Finally, it is important to stress that in order to obtain this result one has to consider not only the manifold $M$, associated to the Euclidean section describing the instanton, but also the related manifold $V$, which is bounded by the sets of fixed points of the Killing vector, associated to isometries in the imaginary time. This should imply boundary contributions also for cosmological, compact solutions.

\section{EULER CHARACTERISTIC AND MANIFOLD STRUCTURE}

The Gauss-Bonnet theorem proves that it is possible to obtain the Euler characteristic of a closed Riemannian manifold $M^{n}$ without boundary from the volume integral of the four-dimensional curvature: 


$$
S_{\mathrm{GB}}=\frac{1}{32 \pi^{2}} \int_{M} \varepsilon_{a b c d} R^{a b} \wedge R^{c d},
$$

where the curvature two-form $R^{a}{ }_{b}$ is defined by the spin connection one-forms $\omega^{a}{ }_{b}$ (for details see the Appendix) as

$$
R_{b}^{a}=d \omega_{b}^{a}+\omega_{c}^{a} \wedge \omega_{b}^{c} .
$$

In a closed Riemannian manifold $M^{n}$, Chern [12,13] has defined the Gauss-Bonnet differential $n$-form $\Omega$ (with $n$ even)

$$
\Omega=\frac{(-1)^{n / 2}}{2^{n} \pi^{n / 2}(n / 2) !} \varepsilon_{a_{1} \cdots a_{n / 2}} R^{a_{1} a_{2}} \wedge \cdots \wedge R^{a_{n+1} a_{n}},
$$

and he has then shown that $\Omega$ can be defined in a manifold $M^{2 n-1}$ formed by the unit tangent vectors of $M^{n}$. In such a way $\Omega$ can be expressed as the exterior derivative of a differential $(n-1)$-form in $M^{2 n-1}$ :

$$
\Omega=-d \Pi \text {. }
$$

He has also proved that the original integral of $\Omega$ over $M^{n}$ can be performed over a submanifold $V^{n}$. This $n$-dimensional submanifold is obtained as the image in $M^{2 n-1}$ of a continuous unit tangent vector field defined over $M^{n}$ with some isolated singular points. By applying Stokes' theorem one thus gets

$$
S_{\mathrm{GB}}^{\mathrm{volume}}=\int_{M^{n}} \Omega=\int_{V^{n}} \Omega=\int_{\partial V^{n}} \Pi .
$$

Since the boundary of $V^{n}$ corresponds exactly to the singular points of the continuous unit tangent vector field defined over $M^{n}$, and bearing in mind that the sum of the indices of a vector field is equal to the Euler characteristic, one finds that the integral of $\Pi$ over the boundary of $V^{n}$ is equal to the Euler number $\chi$. For manifolds with a boundary, this formula can be generalized [14]:

$S_{\mathrm{GB}}=S_{\mathrm{GB}}^{\mathrm{volume}}+S_{\mathrm{GB}}^{\text {boundary }}=\int_{M^{n}} \Omega-\int_{\partial M^{n}} \Pi=\int_{\partial V^{n}} \Pi-\int_{\partial M^{n}} \Pi$.

Thus, the Euler characteristic of a manifold $M^{n}$ vanishes when its boundary coincides with that of a submanifold $V^{n}$ of $M^{2 n-1}$.

The four-manifolds under consideration can have a boundary formed by two disconnected hypersurfaces, say $\partial M^{n}=\left(r_{\text {in }}, r_{\text {out }}\right)$. As far as $V^{n}$ is concerned, the abovequoted unit tangent vector field coincides (again for the cases considered here) with the timelike Killing vector field $\partial / \partial \tau$. Hence the boundary will be the fixed-point sets of such a vector field. The event horizon is always such a set; then the boundaries of $V^{n}$ will be at $r_{h}$ and possibly at one of the actual boundaries of $M^{n}$ which, for sake of simplicity, we shall assume at $r_{\text {out }}$.

\section{ENTROPY FOR MANIFOLDS WITH A BOUNDARY}

Following the definition of gravitational entropy adopted in Ref. [15], we consider a thermodynamical system with conserved charges $C_{i}$ and relative potentials $\mu_{i}$, and we then work in a grand-canonical ensemble. The grand-partition function $Z$, the free energy $W$, and the entropy $S$ are

$$
\begin{gathered}
Z=\operatorname{Tr} \exp \left[-\left(\beta H-\mu_{i} C_{i}\right)\right]=\exp [-W], \\
W=E-T S-\mu_{i} C_{i}, \\
S=\beta\left(E-\mu_{i} C_{i}\right)+\ln Z,
\end{gathered}
$$

respectively. At the tree level of the semiclassical expansion,

$$
\begin{gathered}
Z \sim \exp \left[-I_{E}\right] \\
\left.I_{E}=\frac{1}{16 \pi} \int_{M}\left[(-R+2 \Lambda)+L_{\text {matter }}\right)\right]+\frac{1}{8 \pi} \int_{\partial M}[K],
\end{gathered}
$$

where $I_{E}$ is the on-shell Euclidean action and $[K]=K-K_{0}$ is the difference between the extrinsic curvature of the manifold and that of a reference background. We want to stress here that the procedure we just showed has some subtleties that are well studied in the literature. One of these is that the Schwarzschild solution is a maximum and not a minimum of the Euclidean effective action. This is related to the fact that any black hole in vacuum is a highly unstable object (as can be seen by the negative value of its specific heat $c=-8 \pi M^{2}$ ); but of course any self-gravitating system shows such a behavior, due to the attractive nature of gravity. The problem of performing a thermodynamical analysis of black holes, considering a grand-canonical ensemble, has been studied thoroughly by York [16], who suggested to consider the black hole in a box. Such a choice automatically stabilizes the black hole and enables one to perform a further semiclassical calculation. One can also consider the higherorder corrections to the action. Unfortunately neither oneloop graviton contributions [17] nor matter ones [18] seem to be able to stabilize the black hole, since they are small in comparison with the tree-level term, at least in the regime of negligible back reaction (that is far away from the quantum gravity regime).

To compute $Z$ and $I_{E}$ it is important to correctly take into account the boundaries of the manifold $M^{4}$. We now evaluate separately the two terms occurring in the right-hand side of Eq. (3.4). To obtain $\beta\left(E-\mu_{i} C_{i}\right)$ one can consider the probability of transition between two hypersurfaces at $\tau$ equals constant (where $\tau=i t$ ), say $\tau_{1}$ and $\tau_{2}$. In the presence of conserved charges one gets [15]

$$
\left\langle\tau_{1} \mid \tau_{2}\right\rangle=\exp \left[-\left(\tau_{2}-\tau_{1}\right)\left(E-\mu_{i} C_{i}\right)\right] \approx \exp \left[-I_{E}\right]_{\partial V}
$$

The last equality in this equation is explained by the fact that a hypersurface at $\tau=$ const has a boundary corresponding to the sets of fixed points for the Killing vector $\partial / \partial \tau$. Hence its boundary coincides with that of $V^{n}$.

Remarkably, for the manifolds under consideration, $V_{\text {bulk }}=M_{\text {bulk }}$; therefore the bulk part of the entropy always cancels also for metrics that are not Ricci flat. The entropy then depends on boundary values of the extrinsic curvature only. Thus, one obtains 


$$
S=\beta\left(E-\mu_{i} C_{i}\right)+\ln Z=\frac{1}{8 \pi}\left(\int_{\partial V}[K]-\int_{\partial M}[K]\right) .
$$

The analogy between Eq. (3.6) and Eq. (2.6) is self-evident. For the boundaries of $V$ and $M$, the same considerations as at the end of Sec. II hold.

\section{GRAVITATIONAL ENTROPY AND EULER CHARACTERISTIC FOR SPHERICALLY SYMMETRIC METRICS}

In this section we will find, for a given class of Euclidean spherically symmetric metrics, a general relation between gravitational entropy and Euler characteristic. We will then explicitly treat the most interesting cases.

\section{A. Euler characteristic}

In this section we compute the Euler characteristic for Euclidean spherically symmetric metrics of the form

$$
d s^{2}=e^{2 U(r)} d t^{2}+e^{-2 U(r)} d r^{2}+R^{2}(r) d^{2} \Omega .
$$

The associated spin connections read

$$
\begin{gathered}
\omega^{01}=\frac{1}{2}\left(e^{2 U}\right)^{\prime} d t, \quad \omega^{21}=e^{U} R^{\prime} d \theta, \\
\omega^{31}=e^{U} R^{\prime} \sin \theta d \phi, \quad \omega^{32}=\cos \theta d \phi,
\end{gathered}
$$

and the Gauss-Bonnet action takes the form [9]

$$
\begin{aligned}
S_{\mathrm{GB}}^{\mathrm{volume}} & =\frac{1}{32 \pi^{2}} \int_{M} \varepsilon_{a b c d} R^{a b} \wedge R^{c d}=\frac{1}{4 \pi^{2}} \int_{V} d\left(\omega^{01} \wedge R^{23}\right) \\
& =\frac{1}{4 \pi^{2}} \int_{\partial V} \omega^{01} \wedge R^{23} .
\end{aligned}
$$

The boundary term is $[9,14]$

$$
\begin{aligned}
S_{\mathrm{GB}}^{\text {boundary }} & =-\frac{1}{32 \pi^{2}} \int_{\partial M} \varepsilon_{a b c d}\left(2 \theta^{a b} \wedge R^{c d}-\frac{4}{3} \theta^{a b} \wedge \theta_{e}^{a} \wedge \theta^{e b}\right) \\
& =-\frac{1}{4 \pi^{2}} \int_{\partial M} \omega^{01} \wedge R^{23} .
\end{aligned}
$$

Combining Eqs. (4.3) and (4.4) one eventually gets

$$
S_{\mathrm{GB}}=S_{\mathrm{GB}}^{\text {volume }}+S_{\mathrm{GB}}^{\text {boundary }}=\frac{1}{4 \pi^{2}}\left(\int_{\partial V}-\int_{\partial M}\right) \omega^{01} \wedge R^{23},
$$

where for the metrics $(4.1)$

$$
\begin{gathered}
R^{23}=d \omega^{23}+\omega^{21} \wedge \omega^{13}=\left[1-e^{2 U}\left(R^{\prime}\right)^{2}\right] d \Omega, \\
\omega^{01} \wedge R^{23}=\frac{1}{2}\left(e^{2 U}\right)^{\prime}\left[1-e^{2 U}\left(R^{\prime}\right)^{2}\right] d \Omega d t,
\end{gathered}
$$

and $d \Omega \equiv \sin \theta d \theta d \phi$ is the solid angle.

As already said, we perform our calculations on Riemannian manifolds with compactification of imaginary time,
$0 \leqslant \tau \leqslant \beta$, which is the generalization of the conical singularity removal condition for the metrics under consideration. It is easy to see that this corresponds to choosing ${ }^{1}$

$$
\beta=4 \pi\left[\left(e^{2 U}\right)_{r=r_{h}}^{\prime}\right]^{-1} .
$$

By expressing Eq. (4.5) as a function of the actual boundaries, which are $\partial V^{4}=\left(r_{h}, r_{\text {out }}\right)$ and $\partial M^{4}=\left(r_{\text {in }}, r_{\text {out }}\right)$, one gets

$$
\begin{aligned}
S_{\mathrm{GB}}= & 2\left[1-\left(e^{U} R^{\prime}\right)^{2}\right]_{r_{h}} \\
& -\left[\left(e^{2 U}\right)_{r=r_{h}}^{\prime}\right]^{-1}\left\{\left(e^{2 U}\right)^{\prime}\left[1-\left(e^{U} R^{\prime}\right)^{2}\right]\right\}_{r_{\mathrm{in}}} .
\end{aligned}
$$

We can also rewrite Eq. (4.8) in a more suitable form for our next purposes:

$$
\chi=\frac{\beta}{2 \pi}\left[\left(2 U^{\prime} e^{2 U}\right)\left(1-e^{2 U} R^{\prime 2}\right)\right]_{r_{\text {in }}}^{r_{h}},
$$

expressing the Euler characteristic as a function of the inverse temperature $\beta$.

\section{B. Entropy}

For the metrics (4.1) one can obviously use the general formula (3.6). It is well known [15] that one can write

$$
[K]=\int_{\partial M}\left[\omega^{\mu} n_{\mu}\right],
$$

where for the metrics (4.1) under investigation, $\omega^{\nu}$ and $n_{\nu}$ are

$$
\begin{gathered}
\omega^{\mu}=\left(0,-2 e^{2 U}\left(\partial_{r} U+2 \partial_{r} \ln R\right),-\frac{2 \cot \theta}{r^{2}}, 0\right), \\
n_{\mu}=\left(0, \frac{1}{\sqrt{g^{11}}}, 0,0\right),
\end{gathered}
$$

and they lead to

$$
\omega^{\mu} n_{\mu}=\omega^{1} n_{1}=-2 e^{U}\left(\partial_{r} U+2 \partial_{r} \ln R\right) .
$$

By subtracting from Eq. (4.12) the flat metric correspondent term

$$
d s^{2}=d t^{2}+d r^{2}+r^{2} d \Omega^{2},
$$

one obtains

$$
\begin{gathered}
\omega_{0}^{\mu}=\left(0,-\frac{4}{r},-\frac{2 \cot \theta}{r^{2}}, 0\right), \\
n_{\mu}^{0}=(0,1,0,0),
\end{gathered}
$$

\footnotetext{
${ }^{1}$ Note that condition (4.7) gives an infinite range of time (no period) for extremal black hole metrics [i.e., $\left.\left(e^{2 U}\right)^{\prime}\right|_{r=r_{h}}=0$ ]. This leaves open the question to know if the period of imaginary time remains unfixed or if it has to be infinite, in correspondence to a zero temperature [6].
} 
and

$$
\left[\omega^{\mu} n_{\mu}\right]=\omega^{\mu} n_{\mu}-\omega_{0}^{\mu} n_{\mu}^{0}=-2 e^{U}\left(\partial_{r} U+2 \partial_{r} \ln R\right)+\frac{4}{r} .
$$

Performing the integration of Eq. (3.6) for a spherically symmetric metric, and writing explicitly the dependence on boundaries, one gets

$$
S=-\left.\frac{\beta R}{2}\left[\left(U^{\prime} R+2 R^{\prime}\right) e^{U}-\frac{2 R}{r}\right] e^{U}\right|_{r_{h}} ^{r_{\text {in }}} .
$$

\section{Entropy and topology}

We now prove that a relation between the gravitational entropy and the Euler characteristic can be found for the general case under consideration. One has

$$
\begin{gathered}
A=4 \pi R^{2}\left(r_{h}\right), \\
\beta=4 \pi\left[\left(e^{2 U}\right)_{r=r_{h}}^{\prime}\right]^{-1}, \\
S=\left.\frac{\beta R}{2}\left[\left(U^{\prime} R+2 R^{\prime}\right) e^{U}-\frac{2 R}{r}\right] e^{U}\right|_{r=r_{h}}, \\
\chi=\left.\frac{\beta}{2 \pi}\left(2 U^{\prime} e^{2 U}\right)\left(1-e^{2 U} R^{\prime 2}\right)\right|_{r=r_{h}},
\end{gathered}
$$

hence one can relate $S$ and $\chi$ by their common dependence on $\beta$

$$
\begin{aligned}
S= & \frac{\pi \chi R}{\left(2 U^{\prime} e^{2 U}\right)\left(1-e^{2 U} R^{\prime 2}\right)} \\
& \times\left.\left[\left(U^{\prime} R+2 R^{\prime}\right) e^{2 U}-\frac{2 R}{r} e^{U}\right]\right|_{r=r_{h}} .
\end{aligned}
$$

By definition one has $\left.e^{2 U}\right|_{r=r_{h}}=0$, and Eq. (4.18) then yields

$$
S=\left.\pi \chi R\left(r_{h}\right)\left[\left(e^{2 U}\right)^{\prime}\right]^{-1}\right|_{r=r_{h}}=\frac{\pi \chi R^{2}\left(r_{h}\right)}{2}=\frac{\chi A}{8} .
$$

Some remarks on Eq. (4.19) are in order. We evaluated Eq. (3.6) in a grand-canonical ensemble so this formula a priori is valid only for instantons endowed with nonzero temperature. Nevertheless, as we said, for extremal black holes there is no conical singularity, and therefore no $\beta$ fixing. The fact that Eq. (4.19) gives the expected result also for extremal solutions $^{2}$ [as seen in these cases one gets $\chi=0$, which in Eq. (4.19) straightforwardly gives $S=0$ ] enables us to conjecture that Eq. (4.19) is the general formula, which can be applied to all the known cases of instantons with horizons. The even-

\footnotetext{
${ }^{2} \mathrm{We}$ are referring here to the semiclassical results [6,7] quoted above. For a discussion about the discrepancies with respect to string theory calculations, see the Conclusions.
}

tual lack of intrinsic thermodynamics is simply deducible from Eq. (4.19) by considerations about the topology of the manifold.

We will prove this assumption by studying most of the known solutions with intrinsic thermodynamics. We will start with metrics of the (4.1) form. Moreover, we will show that Eq. (4.19) also holds for an instanton with a Euler characteristic different from 2 (i.e., the Nariai one where the Euler characteristic equals 4) and for an axisymmetric one (i.e., the Kerr metric) which cannot be cast in the form of Eq. (4.1).

We will consider both black hole and cosmological solutions. The formers are asymptotically flat solutions, hence they always have a boundary at infinity, $r_{\text {out }}=\infty$. The inner boundary of $M$ is usually missing since the horizon, after removal of the conical singularity, becomes a regular point of the manifold. By contrast, a drastic change in the boundary structure occurs for extremal black holes. In such a case we cannot fix imaginary time value since metrics present no conical singularity. The horizon is at infinite distance from the external observer; hence it is an inner boundary of $M^{4}$ (i.e., the coordinate of this inner boundary is $r_{\text {in }}$ ).

As far as the cosmological solutions are concerned, they are compact, and therefore $\partial M=0$. Instead, the boundary of $V^{n}$ is only at the horizon that now is also the maximal radius for the space; hence the formulas for the entropy and Euler characteristic are still applicable, setting $r_{\text {out }}=0$ and reversing the sign in front of the equations.

\section{SPHERICALLY SYMMETRIC METRICS}

\section{A. Schwarzschild instanton}

We first consider the Schwarzschild black hole, where

$$
\begin{gathered}
e^{2 U}=(1-2 M / r), \\
U=\frac{1}{2} \ln (1-2 M / r), \\
R=r .
\end{gathered}
$$

Using Eq. (4.7) and bearing in mind that $A=\beta r_{h}=4 \pi r_{h}^{2}$, one can write the relation between $\beta$ and $A$ as

$$
\beta=\frac{A}{r_{h}}
$$

Moreover, from Eq. (4.16) one gets

$$
S=\frac{A}{4},
$$

and from Eq. (4.9) one also finds

$$
\chi=\beta r_{h} \frac{1}{2 \pi r_{h}^{2}}=\frac{A}{2 \pi r_{h}^{2}} .
$$

Now, combining Eqs. (5.3) and (5.4), one obtains

$$
S=\frac{\pi}{2} \chi r_{h}^{2}=\frac{\chi}{32 \pi} \beta^{2}=\frac{\chi A}{8} .
$$




\section{B. Dilaton U(1) black holes}

In the case of the dilaton $\mathrm{U}(1)$ black hole solutions parametrized by $0 \leqslant a \leqslant 1$ (where $a=0$ corresponds to the Reissner-Nordström black hole) one has

$$
\begin{gathered}
e^{2 U}=\left(1-\frac{r_{+}}{r}\right)\left(1-\frac{r_{-}}{r}\right)^{\left(1-a^{2}\right) /\left(1+a^{2}\right)} \\
U=\frac{1}{2} \ln \left[\left(1-\frac{r_{+}}{r}\right)\left(1-\frac{r_{-}}{r}\right)^{\left(1-a^{2}\right) /\left(1+a^{2}\right)}\right], \\
R=r\left(1-\frac{r_{-}}{r}\right)^{a^{2} /\left(1+a^{2}\right)}, \\
M=\frac{r_{+}}{2}+\frac{1-a^{2}}{1+a^{2}} \frac{r_{-}}{2}, \\
Q^{2}=\frac{r_{+} r_{-}}{1+a^{2}}, \\
r_{h}=r_{+} .
\end{gathered}
$$

For such black holes one finds $A=\beta R_{r_{h}}=4 \pi R_{r_{h}}^{2}$, where $R$ determines the characteristic scale of distance. As before [cf. Eq. (5.2)],

$$
\beta=\frac{A}{r_{h}\left(1-r_{-} / r_{h}\right)} .
$$

From Eq. (4.16) one obtains

$$
S=4 \pi R_{r_{h}}^{2}=\frac{A}{4}
$$

and from Eq. (4.9) one finds

$$
\chi=\beta r_{h} \frac{1}{2 \pi R_{h}^{2}}=\frac{A}{2 \pi R_{h}^{2}} .
$$

Again, combining Eqs. (5.8) and (5.9), one gets

$$
S=\frac{\pi}{2} \chi R_{h}^{2}=\frac{\chi A}{8} .
$$

\section{C. de Sitter instanton}

In the de Sitter cosmological case, we can prove how the relation Eq. (4.19) is due to the boundary structure (horizons and "real" boundaries) of the manifold and not to the presence of a black hole. There now is only a cosmological horizon and no proper boundary for $M$, and the topology of the de Sitter instanton is a four-sphere. One has

$$
\begin{gathered}
e^{2 U}=\left(1-\frac{\Lambda}{3} r^{2}\right), \\
U=\frac{1}{2} \ln \left(1-\frac{\Lambda}{3} r^{2}\right), \\
R=r,
\end{gathered}
$$

$$
\begin{gathered}
r_{\Lambda}=\sqrt{\frac{3}{\Lambda}}, \\
A=\frac{12 \pi}{\Lambda}, \\
\beta=2 \pi \sqrt{\frac{3}{\Lambda}},
\end{gathered}
$$

where $r_{\Lambda}$ and $A$ are, respectively, the radius and area of the cosmological horizon. For such compact manifold no Minkowskian subtraction is needed; hence, by using Eq. (4.12) one straightforwardly gets

$$
S=\frac{1}{8 \pi} \int_{\partial V} K
$$

From Eq. (4.15) it is easy to find

$$
\begin{aligned}
\omega^{\mu} n_{\mu} & =-2 e^{U}\left(\partial_{r} U+2 \partial_{r} \ln R\right) \\
& =2\left\{\frac{r \Lambda}{3} \frac{1}{\left[1-\left(r^{2} \Lambda / 3\right)\right]^{1 / 2}}-\frac{2}{r}\left[1-\left(\frac{r^{2} \Lambda}{3}\right)\right]^{1 / 2}\right\} .
\end{aligned}
$$

Hence, bearing in mind Eq. (4.10), one obtains

$$
S=\frac{1}{16 \pi} \int_{r_{\Lambda}} \omega^{\mu} n_{\mu} e^{U} r^{2} \sin \theta d \theta d \tau d \phi=\frac{\beta^{2}}{4 \pi} .
$$

By using Eq. (4.9) with $r_{\text {in }}=0$ and $r_{h}=r_{\Lambda}$, the Euler characteristic is

$$
\chi=\frac{\beta^{2} \Lambda}{6 \pi^{2}}
$$

Combining then Eqs. (5.14) and (5.15), it is easily checked that Eq. (4.19) also holds in the de Sitter case.

\section{Nariai instanton}

The Nariai instanton is the only nonsingular solution of Euclidean vacuum Einstein equation for a given mass $M$ and cosmological constant $\Lambda$. It can be regarded as the limiting case of the Schwarzschild-de Sitter solution when one equals the surface gravity of the black hole to that of the cosmological horizon in order to remove all conical singularities. This could seem meaningless since, in Schwarzschild-de Sitter coordinates, the Euclidean section shrinks to zero (the black hole and cosmological horizons coincide). However, on making an appropriate change of coordinates $[19,20]$, the volume of the Euclidean section no longer vanishes, and the space-time can be properly studied. In this coordinate system, one still deals with a spherically symmetric metric, and the vierbein forms are

$$
e^{0}=\frac{1}{\sqrt{\Lambda}} \sin \xi d \psi, \quad e^{1}=\frac{1}{\sqrt{\Lambda}} d \xi
$$




$$
e^{2}=\frac{1}{\sqrt{\Lambda}} d \theta, \quad e^{3}=\frac{1}{\sqrt{\Lambda}} \sin \theta d \phi
$$

One then obtains

$$
\begin{gathered}
d e^{0}=-\sqrt{\Lambda} \cot \xi e^{0} \wedge e^{1}, \quad d e^{1}=d e^{2}=0, \\
d e^{3}=\sqrt{\Lambda} \cot \theta e^{2} \wedge e^{3},
\end{gathered}
$$

and

$$
\begin{aligned}
& R^{01}=d \omega^{01}=\Lambda e^{0} \wedge e^{1}, \\
& R^{23}=d \omega^{23}=\Lambda e^{2} \wedge e^{3} .
\end{aligned}
$$

Moreover one has

$$
\begin{aligned}
& R=\Lambda^{-1 / 2}, \\
& A=\frac{4 \pi}{\Lambda}, \\
& \beta=\frac{2 \pi}{\sqrt{\Lambda}} .
\end{aligned}
$$

The ranges of integration are $0 \leqslant \psi \leqslant \beta \sqrt{\Lambda}, \quad 0 \leqslant \xi \leqslant \pi$, $0 \leqslant \theta \leqslant 2 \pi, 0 \leqslant \phi \leqslant 2 \pi$. The extremes of $\xi$ correspond to the cosmological horizon and to the black hole horizon [20]. It is worth noting that the period of the imaginary time, $\psi$, is $\beta \sqrt{\Lambda}$, instead of the usual $\beta$. This is due to the normalization of the timelike Killing vector one is forced to choose in this space-time. ${ }^{3}$ The form of the Nariai metric does not enable us to apply Eq. (4.9) and we then compute the Euler characteristic from the very beginning. We obtain

$$
\begin{aligned}
S_{\mathrm{GB}} & =\frac{\Lambda^{2}}{4 \pi^{2}} \int_{V} e^{0} \wedge e^{1} \wedge e^{2} \wedge e^{3} \\
& =\frac{1}{4 \pi^{2}} \int_{0}^{\pi} \sin \xi d \xi \int_{0}^{\beta \sqrt{\Lambda}} d \psi \int_{0}^{\pi} \sin \theta d \theta \int_{0}^{2 \pi} d \phi \\
& =\frac{2 \beta \sqrt{\Lambda}}{\pi} .
\end{aligned}
$$

By substituting $\beta$, one can check that Eq. (5.22) gives the correct result. In fact, the Nariai instanton has topology $S^{2} \times S^{2}$; hence its Euler number, bearing in mind the product formula, is $\chi=2 \times 2=4$.

The entropy can be easily calculated from Eq. (5.12). In this case the extrinsic curvature is given by

$$
K=-\sqrt{\Lambda} \frac{\cos \xi}{\sin \xi}
$$

and one obtains

${ }^{3}$ For a wider discussion of this point, see the Appendix of Ref. [20].

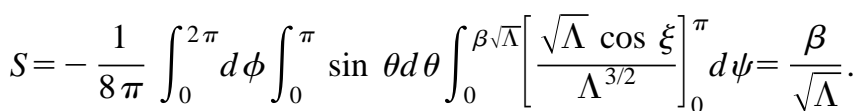

It is now easy to check that the combination of Eqs. (5.22) and (5.24) gives Eq. (4.19).

Remarkably, this implies that Eq. (4.19) cannot be cast in the form

$$
S=\left(\frac{\chi}{2}\right)^{\alpha} \frac{A}{4}
$$

where $\alpha$ could in principle be any positive constant. Since Eq. (4.19) holds also for the Nariai instanton, $\alpha$ must be fixed to 1 .

\section{KERR METRIC}

The Kerr solution describes both the stationary axisymmetric asymptotically flat gravitational field outside a massive rotating body and a rotating black hole with mass $M$ and angular momentum $J$. The Kerr black hole can also be viewed as the final state of a collapsing star, uniquely determined by its mass and rate of rotation. Moreover, its thermodynamical behavior is very different from Schwarzschild or Reissner-Nördstrom black holes, because of its much more complicated causal structure. ${ }^{4}$ Hence its study is of great interest in understanding physical properties of astrophysical objects, as well as in checking any conjecture about thermodynamical properties of black holes.

In terms of Boyer-Lindquist coordinates, the Euclidean Kerr metric reads [22]

$$
\begin{aligned}
d s^{2}= & \frac{\Delta}{\rho^{2}}\left[d t-a \sin ^{2} \theta d \varphi\right]^{2}+\frac{\rho^{2}}{\Delta} d r^{2}+\rho^{2} d \theta^{2} \\
& +\frac{\sin ^{2} \theta}{\rho^{2}}\left[\left(r^{2}+a^{2}\right) d \varphi-a d t\right]^{2},
\end{aligned}
$$

where

$$
\begin{aligned}
& \rho=r^{2}+a^{2} \cos ^{2} \theta, \\
& \Delta=r^{2}-2 M r+a^{2} .
\end{aligned}
$$

Here $a$ is the angular momentum for unit mass as measured from the infinity; it vanishes in the Schwarzschild limit, and $\Delta$ is the Kerr horizon function. The roots of the horizon function $\Delta$ correspond to two null-like surfaces at

$$
r_{ \pm}=M \pm \sqrt{M^{2}-a^{2}}
$$

where $r_{+}$is the Kerr black hole event horizon and $r_{-}$is the Cauchy horizon around the ring singularity at $\rho=0$. The area and the black hole angular velocity are, respectively,

$$
A=4 \pi\left(r_{h}^{2}+a^{2}\right)
$$

\footnotetext{
${ }^{4}$ For instance, Wald pointed out that in a Kerr black hole it is not possible to mimic the Unruh-Rindler case to explain its thermal behavior [21].
} 


$$
\Omega=\frac{a}{\left(r_{h}^{2}+a^{2}\right)}
$$

Such a metric corresponds to the following vierbein forms:

$$
\begin{gathered}
e^{0}=\frac{\sqrt{\Delta}}{\rho}\left(d t-a \sin ^{2} \theta d \varphi\right), \quad e^{1}=\frac{\rho}{\sqrt{\Delta}} d r, \\
e^{2}=\rho d \theta, \quad e^{3}=\frac{\sin \theta}{\rho}\left[\left(r^{2}+a^{2}\right) d \varphi-a d t\right],
\end{gathered}
$$

where $\rho$ is the positive square root of $\rho^{2}$.

From Eq. (6.6), one can obtain the spin connection oneforms as [22]

$$
\begin{gathered}
\omega^{0}{ }_{1}=-\frac{a r \sin \theta}{\rho^{3}} e^{3}+F e^{0}, \\
\omega_{2}^{0}=-\frac{a \cos \theta \sqrt{\Delta}}{\rho^{3}} e^{3}-a^{2} \frac{\sin \theta \cos \theta}{\rho^{3}} e^{0}, \\
\omega_{3}^{0}=-\frac{a r \sin \theta}{\rho^{3}} e^{1}+\frac{a \cos \theta \sqrt{\Delta}}{\rho^{3}} e^{2}, \\
\omega^{1}{ }_{2}=-a^{2} \frac{\sin \theta \cos \theta}{\rho^{3}} e^{1}-r \frac{\sqrt{\Delta}}{\rho^{3}} e^{2}, \\
\omega^{3}{ }_{1}=-\omega^{1}{ }_{3}=r \frac{\sqrt{\Delta}}{\rho^{3}} e^{3}+\frac{a r \sin \theta}{\rho^{3}} e^{0}, \\
\omega_{3}^{2}=-\frac{\cos \theta}{\sin \theta} \frac{\left(r^{2}+a^{2}\right)}{\rho^{3}} e^{3}-\frac{a}{\rho^{3}} \cos \theta \sqrt{\Delta} e^{0},
\end{gathered}
$$

where [22]

$$
F \equiv \frac{\partial}{\partial r} \frac{\sqrt{\Delta}}{\rho}=\frac{(r-M) \rho^{2}-r \Delta}{\rho^{3} \sqrt{\Delta}} .
$$

By virtue of Eqs. (6.8) and (2.2), and the nilpotency of the exterior derivative operator $d$, the Gauss-Bonnet action in Eq. (2.6) takes the form

$$
\begin{aligned}
S_{\mathrm{GB}}= & -\frac{1}{4 \pi^{2}} \int\left(\omega^{01} \wedge R^{23}+\omega^{02} \wedge \omega^{21} \wedge \omega^{23}+\omega^{03} \wedge \omega^{31} \wedge \omega^{23}\right. \\
& \left.+\omega^{02} \wedge d \omega^{31}\right)_{r_{h}},
\end{aligned}
$$

where $d \omega^{31}$ can be expressed in terms of a suitable combination (wedge product) of the type $e^{i} \wedge e^{j}$, and $r_{h}$ is the radius of the Kerr horizon (i.e., the positive roots of $\Delta=0$ ). For further details see the Appendix and Ref. [22]. By defining the quantities [22]

$$
\begin{gathered}
I \equiv \frac{M r}{\rho^{6}}\left(r^{2}-3 a^{2} \cos ^{2} \theta\right), \\
K \equiv \frac{M a \cos \theta}{\rho^{6}}\left(3 r^{2}-a^{2} \cos ^{2} \theta\right),
\end{gathered}
$$

one obtains, for the quantity in parentheses, in Eq. (6.10),

$$
\begin{aligned}
& e^{0} \wedge e^{2} \wedge e^{3}\left(2 F I \frac{3 r a^{4}(\sin \theta \cos \theta)^{2} \sqrt{\Delta}}{\rho^{9}}\right) \\
& -e^{0} \wedge e^{1} \wedge e^{3}\left(\frac{8 M r^{3} a^{2} \sin \theta \cos \theta}{\rho^{9}}\right),
\end{aligned}
$$

in terms of one-forms appearing in Eq. (6.6). Such a quantity has to be evaluated at $r=r_{h}$.

At this stage some remarks are in order. In the Euclidean path-integral approach the Kerr solution is an instanton (i.e., a nonsingular solution of the Euclidean action) only after the identification of the points $(\tau, r, \theta, \varphi)$ and $\left(\tau+2 \pi \kappa_{1}^{-1}, r, \theta, \varphi+2 \pi \kappa_{1}^{-1} \kappa_{2}\right)$ [5], where $\kappa_{1}=\kappa$ is the surface gravity of the black hole and $\kappa_{2}= \pm \Omega$. With this identification, the Euclidean section has topology $R^{2} \times S^{2}$ and $\chi=2$. The condition of a periodic isometry group implies $\kappa_{2} / \kappa_{1}=q$ [8], where $q \in Q$ is a rational number. By using this relation, it is easy to see that the periods are

$$
\begin{gathered}
\beta_{\tau}=2 \pi \kappa_{1}=4 \pi \frac{M r_{h}}{\sqrt{\left(M^{2}-a^{2}\right)}}, \\
\beta_{\varphi}=2 \pi \frac{\kappa_{1}}{k_{2}}=2 \pi q,
\end{gathered}
$$

If one would set $q \neq 1$, Eq. (3.6) for the black hole entropy would acquire a factor $q$, but this spurious factor would be absorbed in the change of the period of $\varphi$ that implies a redefinition of the black hole area (6.5), which would become $A=4 \pi q\left(r_{h}^{2}+a^{2}\right)$. Therefore one still expects $S=A / 4$, and the fixing of $q=1$ will not bring about a loss of generality. Moreover in this way the area will be the "physical", one, as written in Eq. (6.5). Hence the Euler number is

$$
\begin{aligned}
\chi= & \frac{M r_{h}\left(r_{h}-M\right)}{4 \pi^{2}} \int_{0}^{\beta} d \tau \int_{0}^{2 \pi} d \varphi \int_{0}^{\pi} \frac{\left(r_{h}^{2}-3 a^{4} \cos ^{4} \theta\right)}{\left(r_{h}^{2}+a^{2} \cos ^{2} \theta\right)^{3}} \\
& \times \sin \theta d \theta=\frac{2}{\pi} \beta\left(r_{h}-M\right) \frac{M r_{h}}{\left(r_{h}^{2}+a^{2}\right)^{2}} .
\end{aligned}
$$

Bearing in mind Eq. (6.12) and that $\left(r_{h}^{2}+a^{2}\right)=2 M r_{h}$, one eventually gets

$$
\chi=8 \frac{M^{2} r_{h}^{2}}{\left(r_{h}^{2}+a^{2}\right)^{2}}=2 .
$$

As far as the entropy is concerned, here we follow the procedure outlined in Secs. III and IV B. From Eq. (3.6), writing $\omega^{\mu}$ as

$$
\omega^{\mu}=-\frac{2}{\sqrt{g}}\left(\frac{\partial \sqrt{g}}{\partial x^{\nu}}\right) g^{\mu \nu}-\frac{\partial g_{\nu \mu}}{\partial x^{\nu}},
$$

and bearing in mind that the Kerr determinant is

$$
\sqrt{g}=\rho^{2} \sin \theta
$$

one finds 


$$
\begin{gathered}
\omega^{\mu}=\left(0,-2 \frac{r \Delta}{\rho^{4}},-\frac{2(r-M)}{\rho^{2}},-2 \frac{\cot \theta}{\rho^{2}}, 0\right), \\
n_{\mu}=\left(0, \frac{\rho}{\sqrt{\Delta}}, 0,0\right) .
\end{gathered}
$$

By subtracting the flat Minkowskian term of $\omega^{\mu}$ [see Eq. (5.7)] one easily obtains

$$
\left[\omega^{\mu} n_{\mu}\right]=-\frac{2}{\rho \sqrt{\Delta}}\left(\frac{r \Delta}{\rho^{2}}+r-M\right)+\frac{4}{r} .
$$

One can then evaluate the Kerr black hole entropy:

$$
\begin{aligned}
S= & -\frac{1}{16 \pi} \int_{0}^{\beta} d \tau \int_{0}^{\pi} d \varphi \int_{0}^{2 \pi} d \theta \rho \sqrt{\Delta} \\
& \times \sin \theta \cdot\left[-\frac{2}{\rho \sqrt{\Delta}}\left(\frac{r \Delta}{\rho^{2}}+r-M\right)+\frac{4}{r}\right]_{r_{h}}=\frac{\beta}{2}\left(r_{h}-M\right) .
\end{aligned}
$$

Thus, combining Eqs. (6.13) and (6.19), one has

$$
S=\frac{\pi}{4} \frac{\left(r_{h}^{2}+a^{2}\right)^{2}}{M r_{h}} \chi=\frac{1}{2} \pi\left(r_{h}^{2}+a^{2}\right) \chi=\frac{A}{8} \chi .
$$

\section{CONCLUSIONS}

The main result of this work is a new formulation of the Bekenstein-Hawking entropy. This has been achieved by making explicit how gravitational entropy depends on topology. This result has been proved to be valid for a wide class of gravitational instantons endowed with intrinsic thermodynamics. Therefore, it can be considered a confirmation and generalization of previous results $[10,11]$ (obtained for black holes and in a different formalism) as well as a compact, general formulation of the Bekenstein-Hawking relation.

Although our results seem to imply a central role for space-time topology in the explanation of intrinsic thermodynamics of gravitational instantons, we are not claiming that it is not necessary to understand the microscopic degrees of freedom of black holes in order to understand their entropy. The fact that the horizon's area is still present in Eq. (4.19) implies a dependence for the gravitational entropy on two different objects: a discrete topological parameter (i.e., the Euler characteristic, which can be 0,2 , or 4 for the known solutions with event horizons) and the area, which can vary with continuity. Since the topological term comes out in the general form (4.19), for all the space-times endowed with intrinsic thermodynamics, this seems to imply that the topological nontriviality of space-time is a necessary (although probably not sufficient) condition for the coming up of the, otherwise hidden, microscopic nature of gravity. The origin of these hidden degrees of freedom is still a matter of debate. The authors suggest that the relation here clearly shown between the entropy and the boundary structure of the four-manifold seems to add evidence in favor of an interpretation based on the dynamical degrees of freedom associated to vacuum in topological nontrivial four- manifolds [23]. This enables us to argue that intrinsic thermodynamics of some gravitational instantons could be due to a sort of "gravitational Casimir effect" [24] on such fourmanifolds.

Finally we stress that the interesting results $[25,26]$, about the nonzero entropy of some extremal string theory black hole solutions, are not necessarily in contrast with ours. In fact recent calculations $[27,28]$ have proved that the discrepancies between semiclassical and string theory results can be eliminated if one performs in the former approach a sum over topologies and imposes the extremality condition after quantization. It is easy to see [28] that with this procedure the nontrivial topologies will dominate, in this case reducing the case to a nonextremal one, with nonzero entropy. Hence it seems that string model results implicitly involve a quantization procedure where the classical extremal topology is ignored by means of the quantization procedure. Of course it is still an open question which of the two procedures could better fit reality.

In the opinion of the authors all these problems are deeply intertwined and hence they deserve further investigation.

\section{ACKNOWLEDGMENTS}

S. Liberati wishes to thank F. Belgiorno for illuminating remarks and M. Maggiore and M. Martellini for constructive advice. G. Pollifrone thanks D. Bellisai for correspondence about Kerr black holes. Both authors are also grateful to P. Blaga, G. Esposito, R. Garattini, G. Immirzi, B. Jensen, and K. Yoshida for extensive discussions. The work of G. Pollifrone was supported in part by the Angelo Della Riccia Foundation.

\section{APPENDIX}

The Euler number of a four-manifold can be defined as an alternating sum of Betti numbers:

$$
\chi \equiv \sum_{n=0}^{4}(-1)^{n} B_{n} .
$$

The $n$th Betti number $B_{n}$ is the number of independent closed $n$-surfaces that are not boundaries of some $(p+1)$-surface. For a compact manifold without boundary, $B_{n}$ is also equal to the number of linear independent harmonic $n$-forms, and $B_{n}=B_{4-n}$ (i.e., $B_{0}=B_{4}=1$ and $B_{1}=B_{3}$ ). If the four-manifold is simply connected, $B_{1}=0$, whereas if there is a boundary, $B_{0}=1$ and $B_{4}=0$.

In the Cartan approach to geometry one deals with differential forms (see Sec. II). Defining a local coordinate basis of one-forms $d x^{\mu}$ and a local orthonormal basis of one-forms $e^{a}$ over a four-manifold $M$, the metric can be expressed as

$$
g=g_{\mu \nu} d x^{\mu} \otimes d x^{\nu}=\eta_{a b} e^{a} \otimes e^{b},
$$

where $\eta_{a b}$ is the flat Euclidean metric tensor with signature +4 , and the information about the curvature of the Riemannian four-space is encoded in

$$
e^{b}=e_{\mu}^{b} d x^{\mu} .
$$


Here $e_{\mu}^{a}(x)$ are the vierbein (or tetrad) one-forms, and they can be viewed as a sort of square root of the metric. Note that the Greek letters $\mu, \nu, \ldots$ denote abstract indices, and Latin letters $a, b, \ldots$ internal indices.

We can now introduce the spin connection one-forms $\omega^{a}{ }_{b}$ and define the first Cartan structure equation:

$$
T^{a} \equiv d e^{a}+\omega^{a}{ }_{b} \wedge e^{b}=\frac{1}{2} T^{a}{ }_{b c} e^{b} \wedge e^{c},
$$

where $T^{a}$ is the torsion two-form of the manifold. The second Cartan structure equation defines the curvature two-form of the manifold [see Eq. (2.1)]:

$$
R_{b}^{a} \equiv d \omega_{b}^{a}+\omega_{c}^{a} \wedge \omega_{b}^{c}=\frac{1}{2} R_{b c d}^{a} e^{c} \wedge e^{d}
$$

In the tensor formalism the covariant derivative $\nabla_{\alpha}$ is defined by using the Levi-Civita connection (or Christoffel symbols) $\Gamma_{\mu \nu}^{\alpha}$. By virtue of the metricity conditions (i.e., $\nabla_{\alpha} g_{\mu \nu}=0$ ) and of the absence of torsion (i.e., $T_{[\alpha \beta]}^{\mu}=0$ ), the Levi-Civita connection is then uniquely determined in terms of the metric. In the Cartan approach, the spinconnection one-forms replace the Christoffel symbols. The Levi-Civita spin-connection one-forms are then obtained by imposing the metricity and the torsionless conditions, which yield

$$
\omega_{a b}=-\omega_{b a}
$$

and

$$
d e^{a}+\omega^{a}{ }_{b} \wedge e^{b}=0
$$

respectively.

In Sec. VI the term $\varepsilon_{a b c d} R^{a b} \wedge R^{c d}$ occurring in the GaussBonnet action (6.10) reads

$$
\begin{aligned}
\varepsilon_{a b c d} R^{a b} \wedge R^{c d}= & 6\left[d \left(\omega^{01} \wedge R^{23}+\omega^{02} \wedge \omega^{21} \wedge \omega^{23}\right.\right. \\
& \left.+\omega^{03} \wedge \omega^{31} \wedge \omega^{23}+\omega^{02} \wedge d \omega^{31}\right) \\
& \left.+d \omega^{03} \wedge d \omega^{12}\right] .
\end{aligned}
$$

The last term $d \omega^{03} \wedge d \omega^{12}=d\left(\omega^{03} \wedge d \omega^{12}\right)$, bearing in mind that $e^{i} \wedge e^{i}=0$, vanishes by virtue of the structure of $\omega^{03}$ and $\omega^{12}$ [see Eq. (6.6)]. Furthermore, the term $d \omega^{31}$ takes the form

$$
\begin{aligned}
d \omega^{31}= & -\frac{a \sin \theta}{\rho^{3}}\left(\frac{r F+\sqrt{\Delta}}{\rho^{3}} a^{2} \cos ^{2} \theta\right) e^{0} \wedge e^{1} \\
& -\frac{a r \cos \theta}{\rho^{6}}\left(\rho^{2}+2 a^{2} \sin ^{2} \theta\right) e^{0} \wedge e^{2} \\
& +\frac{1}{\rho^{6}}\left[\Delta\left(\rho^{2}-2 r^{2}\right)+r \rho^{2}(r-M)\right] e^{1} \wedge e^{3} \\
& +\frac{r \sqrt{\Delta} \cos \theta}{\rho^{6} \sin \theta}\left(\rho^{2}-a^{2} \sin ^{2} \theta\right) e^{2} \wedge e^{3} .
\end{aligned}
$$

[1] J. M. Bardeen, B. Carter, and S. W. Hawking, Commun. Math. Phys. 31, 161 (1973).

[2] J. D. Bekenstein, Phys. Rev. D 7, 2333 (1973).

[3] D. Christodoulou and R. Ruffini, Phys. Rev. D 4, 3552 (1971).

[4] S. W. Hawking, Commun. Math. Phys. 43, 99 (1975).

[5] G. W. Gibbons and S. W. Hawking, Phys. Rev. D 15, 2752 (1977).

[6] S. W. Hawking, G. T. Horowitz, and S. F. Ross, Phys. Rev. D 51, 4302 (1995).

[7] S. W. Hawking and G. T. Horowitz, Class. Quantum Grav. 13, 1487 (1996).

[8] G. W. Gibbons and S. W. Hawking, Commun. Math. Phys. 66, 291 (1979).

[9] G. W. Gibbons and R. E. Kallosh, Phys. Rev. D 51, 2839 (1995).

[10] M. Banados, C. Teitelboim, and J. Zanelli, Phys. Rev. Lett. 72, 957 (1994).

[11] C. Teitelboim, Phys. Rev. D 51, 4315 (1995).

[12] S. Chern, Ann. Math. 45, 747 (1944).

[13] S. Chern, Ann. Math. 46, 674 (1945).

[14] T. Eguchi, P. B. Gilkey, and A. J. Hanson, Phys. Rep. 66, 6 (1980).
[15] R. E. Kallosh, T. Ortin, and A. Peet, Phys. Rev. D 47, 5400 (1993).

[16] J. W. York, Phys. Rev. D 33, 2092 (1986).

[17] D. J. Gross, M. J. Perry, and L. G. Yaffe, Phys. Rev. D 25, 330 (1982).

[18] F. Belgiorno and S. Liberati, Phys. Rev. D 54, 3172 (1996).

[19] P. Ginsparg and M. J. Perry, Nucl. Phys. B222, 245 (1983).

[20] R. Bousso and S. W. Hawking, Phys. Rev. D 54, 6312 (1996).

[21] R. M. Wald, Quantum Field Theory in Curved Space-Time and Black Hole Thermodynamics (University of Chicago Press, Chicago, 1994).

[22] B. O'Neill, The Geometry of Kerr Black Holes (A.K. Peters, Wellesley, MA, 1995).

[23] S. Hacyan, A. Sarmiento, G. Cocho, and F. Soto, Phys. Rev. D 32, 914 (1985).

[24] F. Belgiorno and S. Liberati, "Black Hole Thermodynamics, Casimir Effect and Induced Gravity,' Report No. SISSA-178/ 96/A, gr-qc/9612024.

[25] A. Sen, Mod. Phys. Lett. A 10, 2081 (1995).

[26] A. Strominger and C. Vafa, Phys. Lett. B 379, 99 (1996).

[27] A. Ghosh and P. Mitra, Phys. Rev. Lett. 77, 4848 (1996).

[28] A. Ghosh and P. Mitra, Phys. Rev. Lett. 78, 1858 (1997). 